\title{
Constructing the Second Order Poincaré Map Based on the Hopf-Zero Unfolding Method
}

\author{
Gen $\mathrm{Ge}^{1}$ and Wang Wei ${ }^{2}$ \\ ${ }^{1}$ School of Mechanical Engineering, Tianjin Polytechnic University, Tianjin 300072, China \\ ${ }^{2}$ Department of Mechanics, Tianjin University, Tianjin 300072, China \\ Correspondence should be addressed to Gen Ge; gegenroot@126.com
}

Received 12 August 2013; Revised 12 September 2013; Accepted 13 September 2013

Academic Editor: Massimiliano Ferrara

Copyright (c) 2013 G. Ge and W. Wei. This is an open access article distributed under the Creative Commons Attribution License, which permits unrestricted use, distribution, and reproduction in any medium, provided the original work is properly cited.

\begin{abstract}
We investigate the Shilnikov sense homoclinicity in a 3D system and consider the dynamical behaviors in vicinity of the principal homoclinic orbit emerging from a third order simplified system. It depends on the application of the simplest normal form theory and further evolution of the Hopf-zero singularity unfolding. For the Shilnikov sense homoclinic orbit, the complex form analytic expression is accomplished by using the power series of the manifolds surrounding the saddle-focus equilibrium. Then, the second order Poincaré map in a generally analytical style helps to portrait the double pulse dynamics existing in the tubular neighborhood of the principal homoclinic orbit.
\end{abstract}

\section{Introduction}

In recent years, there has been a great deal interest in understanding the dynamics and bifurcations in problems governed by $3 \mathrm{D}$ autonomous differential systems [1-3]. These problems include sequences of periodic doubling, existence of strange attractors, and bifurcations of the multipulse orbits. The common adapted tool for analyzing multipulse behaviors of the 3D nonlinear dynamical system is the well-known Poincaré map [4]. It starts by a standard analysis of the local and global cross-sections to the principal homoclinic orbit of the equilibrium, where the stable and unstable manifolds can intersect along the tubular neighborhood of the principal homoclinic loop, giving rise to Poincaré homoclinic structure. Among the literature, Glendinning and Sparrow [5] studied the local behavior of systems' near homoclinic orbits to the stationary equilibrium of saddle-focus type. They described the process of periodic orbit approached homoclinicity and discussed the resulting global patterns of bifurcations, such as the emergency of subsidiary homoclinic manifolds. More explicit work is offered by Gonchenko et al. [6]. They reported the research of multipulse homoclinic loops in two-parameter families of vector fields surrounding the principal homoclinic tangency and then proved the high structural instability in the neighborhood of a Shilnikov type saddle-focus.

The prosperities of the Poincaré map reflect the topological and stability of the actual flow and are basically adapted in this paper to concern the dynamics of doublepulse orbit at the tubular field. Besides that we complexly quantify the principal orbit before the analysis of the return map with the expectation to generalize the expression and obtain higher computational precise. Actually, the so-called principal homoclinicity has been frequently mentioned [79], but the orbit itself and its usage for the return map have not been broadly detected. Li and Zhu constructed the $s$ dimensional series expression of the stable and unstable manifolds [10], which formed the homoclinic orbit near the hyperbolic singular point of the Lorenz system.

With a further extension of the methodology, the Shilnikov sense principal homoclinic loop will be analytically obtained from the composition of series expression of the invariant manifolds surrounding the saddle-focus equilibrium. These quantified manifolds intersect the cross-sections locally and globally produce more explicit coordinates up to a desired order and also characterize the Poincaré homoclinic map of the system. 
When talking about the complex dynamics, we acknowledge the relevance of the splitting homoclinic connection with the unfolding of the Hopf-zero singularity [11]. Meanwhile, the local dynamics presented by a normal form approach (NF) [12-15], a standard result of Birkhoff NF theory, is also applied in the initial simplification.

So, in this paper, we try to construct a second order Poincaré map based on the Hopf-zero unfolding in a common 3D system. In Section 2, it is supposed that the system exhibit the Hopf-zero singularity at the fixed parameter value $\mu=0$ of the linear part of the vector field. In Section 3, the explicit expression of the principal homoclinic loop $H$ is given by using the invariant manifold method. In Section 4, analytical series expressions motivate our interest in chasing a more general and higher order Poincaré map from its quantified stable and unstable manifolds. The new map can be easily degenerated to the original result presented by Wiggins [16]. And it gives further facility of studying the complex dynamics, such as subsidiary or multipulse homoclinicity surrounding the principal homoclinic orbit.

\section{The Shilnikov Unfolding Based on the Hopf- Zero Normal Form}

Consider the following 3D system:

$$
\begin{aligned}
\dot{x}= & y+f(x, y, z ; \mu), \\
\dot{y}= & z+g(x, y, z ; \mu), \\
\dot{z}= & \varepsilon_{1} x+\varepsilon_{2} y+\varepsilon_{3} z+h(x, y, z ; \mu) \\
& (x, y, z, \mu) \in \mathbb{R}^{1} \times \mathbb{R}^{1} \times \mathbb{R}^{1} \times \mathbb{R}^{1},
\end{aligned}
$$

where $\mu$ is regarded as the orbit splitting parameter and $f_{1}, f_{2}, f_{3}$ are $C^{r}, r \geq 2$ and vanish along with their derivates at $(x, y, z, \mu)=(0,0,0,0)$. As mentioned in [11], the unfolding of the Hopf-zero local bifurcation contains the global Shilnikov sense homoclinicity emerging from the saddle-focus equilibrium. Given that (1) enjoys Hopf-zero singularity near the origin, the normal form (NF) can be written as

$$
\begin{aligned}
\dot{u}_{1}= & (\gamma+i \omega) u_{1}+a_{1101} u_{1} u_{2} \\
& +u_{1} \sum_{p=1}^{m_{1}} \sum_{j=0}^{p} a_{1, p-j+1, p-j, 2 j}\left(\left|u_{1}\right|^{2}\right)^{p-j} u_{2}^{2 j} \\
& +u_{1} \sum_{p=1}^{m_{2}} \sum_{j=0}^{p} a_{1, p-j+1, p-j, 2 j+1}\left(\left|u_{1}\right|^{2}\right)^{p-j} u_{2}^{2 j+1},
\end{aligned}
$$

$$
\begin{aligned}
\dot{u}_{2}= & \lambda u_{2}+\sum_{p=1}^{m_{3}} \sum_{j=0}^{p} a_{2, p-j, p-j, 2 j}\left(\left|u_{1}\right|^{2}\right)^{p-j} u_{2}^{2 j} \\
& +\sum_{p=1}^{m_{1}} \sum_{j=0}^{p} a_{2, p-j, p-j, 2 j+1}\left(\left|u_{1}\right|^{2}\right)^{p-j} u_{2}^{2 j+1}
\end{aligned}
$$

in which $\left|u_{1}\right|^{2}=u_{1} \bar{u}_{1}$, an over-bar denotes complex conjugate; for example, $\bar{u}_{1}$ denotes the complex conjugate of $u_{1} ; a_{1, \kappa}$ and $a_{2, \kappa}$ are the NF coefficients, given in the complex and real forms, respectively. The final expressions have different forms depending on whether the total order $n$ of the NF is an odd or even number; that is,

(i) $m_{1}=m_{2}+1=m_{3}=(1 / 2)(n-1)$, if $n$ is odd;

(ii) $m_{1}=m_{2}=m_{3}-1=(n / 2)-1$, if $n$ is even.

Here we only use the third order NF in (2) because the system is terminated in the third order [11]:

$$
\begin{aligned}
& \dot{u}_{1}=(\gamma+\omega i) u_{1}+a_{1101} u_{1} u_{2}+a_{1102} u_{1} u_{2}^{2}+a_{1210} u_{1}^{2} \bar{u}_{1}, \\
& \dot{u}_{2}=\lambda u_{2}+a_{2002} u_{2}^{2}+a_{2110} u_{1} \bar{u}_{1}+a_{2111} u_{1} \bar{u}_{1} u_{2}+a_{2003} u_{2}^{3} .
\end{aligned}
$$

The dynamics of bifurcation near the structurally unstable homoclinic orbits in the Shilnikov sense requires the description on a two-parameter group $(\mu, \rho)$. It takes the function as the distance between the point of the intersection of principal homoclinic $H$ with some surface of the crosssection and the line of intersection of stable manifold $W^{s}$ with the same surface of section. On the other hand, $\rho=\gamma+\omega i / \lambda$, defined in its complex form. Further discussion is restricted to the condition $\left|\rho_{r}=\gamma / \lambda\right|<1$.

Firstly, it is needed to quantify the analytical structure of the principal homoclinic orbit in the complex form at $\mu=$ 0 . And then constructing a second order Poincaré map to prohibit a clear insight of the complex dynamics surrounding the principal orbit $H$ is carried out.

\section{Analytical Expression of the Principal Homoclinic Orbit}

The analytical expression of the principal homoclinic orbit is the combination of the $1 \mathrm{D}$ manifold associated with the eigenvalue $\lambda_{1}=\lambda$ and the $2 \mathrm{D}$ manifold associated with $\lambda_{2,3}=\gamma \pm \omega i$. So, the $1 \mathrm{D}$ manifold for (3) is

$$
\begin{aligned}
& u_{1}=\sum_{m=0}^{k} \Gamma_{m} e^{m \lambda t}, \quad \bar{u}_{1}=\sum_{m=0}^{k} \bar{\Gamma}_{m} e^{m \lambda t} \\
& u_{2}=\sum_{m=0}^{k} \Delta_{m} e^{m \lambda t}
\end{aligned}
$$


where $\Gamma_{m}, \bar{\Gamma}_{m}$, and $\Delta_{m}$ are the coefficients to be determined. $\Gamma_{m}$ is in the complex form and $\bar{\Gamma}_{m}$ denotes its complex conjugate. The $\Delta_{m}$ is a real number. Differentiate (4) with respect to time $t$ and substitute it into (3) and then balance the same order terms of $e^{m \lambda t}$ on both sides of the resulting equations. That yields

$$
\begin{aligned}
& \Gamma_{k}=- \frac{1}{\gamma-k \lambda+i \omega} \\
& \times\left(a_{1101} \sum_{m=0}^{k} \Gamma_{m} \Delta_{k-m}\right. \\
&+a_{1102} \sum_{m=0}^{k} \sum_{j=0}^{k-m} \Gamma_{m} \Delta_{j} \Delta_{k-m-j} \\
&\left.+a_{1210} \sum_{m=0}^{k} \sum_{j=0}^{k-m} \Gamma_{m} \Gamma_{j} \bar{\Gamma}_{k-m-j}\right), \\
& \bar{\Gamma}_{k}=\frac{1}{-\gamma+}+k \lambda+i \omega \\
& \times\left(\bar{a}_{1101} \sum_{m=0}^{k} \bar{\Gamma}_{m} \Delta_{k-m}\right. \\
&+\bar{a}_{1102} \sum_{m=0}^{k} \sum_{j=0}^{k-m} \bar{\Gamma}_{m} \Delta_{j} \Delta_{k-m-j} \\
&\left.+\bar{a}_{1210} \sum_{m=0}^{k} \sum_{j=0}^{k-m} \bar{\Gamma}_{m} \bar{\Gamma}_{j} \Gamma_{k-m-j}\right),
\end{aligned}
$$

$$
\begin{aligned}
& \Delta_{k}=\frac{1}{(-1+k) \lambda} \\
& \quad \times\left(a_{2002} \sum_{m=0}^{k} \Delta_{k-m} \Delta_{m}\right. \\
& \quad+a_{2110} \sum_{m=0}^{k} \Gamma_{m} \bar{\Gamma}_{k-m} \\
& \quad+a_{2111} \sum_{m=0}^{k} \sum_{j=0}^{k-m} \Gamma_{m} \bar{\Gamma}_{j} \Delta_{k-m-j} \\
& \left.\quad+a_{2003} \sum_{m=0}^{k} \sum_{j=0}^{k-m} \Delta_{m} \Delta_{j} \Delta_{k-m-j}\right) .
\end{aligned}
$$

The 2D manifolds subject to the complex enginvalues $\lambda_{2,3}$ are

$$
\begin{aligned}
u_{1}= & \eta+h_{1,2}(\eta, \bar{\eta})+h_{1,3}(\eta, \bar{\eta})+\cdots+h_{1, k}(\eta, \bar{\eta}), \\
\bar{u}_{1}= & \bar{\eta}+\bar{h}_{1,2}(\eta, \bar{\eta})+\bar{h}_{1,3}(\eta, \bar{\eta})+\cdots+\bar{h}_{1, k}(\eta, \bar{\eta}), \\
u_{2}= & \Delta_{1,0} \eta+\Delta_{0,1} \bar{\eta}+h_{2,2}(\eta, \bar{\eta})+h_{2,3}(\eta, \bar{\eta}) \\
& +\cdots+h_{2, k}(\eta, \bar{\eta}),
\end{aligned}
$$

where $h_{1, j}(\eta, \bar{\eta})=\sum_{i=0}^{j} \Gamma_{i, j-i} \eta^{i} \bar{\eta}^{j-i}, h_{2, j}(\eta, \bar{\eta})=\sum_{i=0}^{j} \Delta_{i, j-i} \eta^{i} \bar{\eta}^{j-i}$, $j=2, \ldots, k$, and $\Gamma_{m, n}, \bar{\Gamma}_{m, n}, \Delta_{m, n}$ are the coefficients to be determined, $n=k-m \cdot \eta$, $\bar{\eta}$ can be expressed in the following complex form: $\eta=\left(\Gamma_{r, 1,0}+i \Gamma_{i, 1,0}\right) e^{\lambda_{2} t}, \bar{\eta}=\left(\Gamma_{r, 1,0}-i \Gamma_{i, 1,0}\right) e^{\lambda_{3} t}$. Differentiate (6) with respect to time $t$ and substitute it into (3) and then balance the same order terms of $e^{\left(m \lambda_{2} t+n \lambda_{3} t\right)}$ on both sides of the resulting equation. It gives the coefficients of the 2D manifold:

$$
\begin{aligned}
\Gamma_{m, n}=\frac{1}{(-1+m+n) \gamma+i(-1+m-n) \omega}( & a_{1101} \sum_{m_{1}=0}^{m} \sum_{n_{1}=0}^{n} \Gamma_{m_{1}, n_{1}} \Delta_{m-m_{1}, n-n_{1}} \\
& +a_{1102} \sum_{m_{1}=0}^{m} \sum_{m_{2}=0}^{m-m_{1}} \sum_{n_{1}=0}^{n} \sum_{n_{2}=0}^{n-n_{1}} \Gamma_{m_{1}, n_{1}} \Delta_{m_{2}, n_{2}} \Delta_{m-m_{1}-m_{2}, n-n_{1}-n_{2}} \\
& \left.+a_{1210} \sum_{m_{1}=0}^{m} \sum_{m_{2}=0}^{m-m_{1}} \sum_{n_{1}=0}^{n} \sum_{n_{2}=0}^{n-n_{1}} \Gamma_{m_{1}, n_{1}} \Gamma_{m_{2}, n_{2}} \bar{\Gamma}_{n-n_{1}-n_{2}, m-m_{1}-m_{2}}\right) \\
\bar{\Gamma}_{m, n}=\frac{1}{(-1+m+n) \gamma-i(-1+m-n) \omega}( & \bar{a}_{1101} \sum_{m_{1}=0}^{m} \sum_{n_{1}=0}^{n} \bar{\Gamma}_{m_{1}, n_{1}} \Delta_{n-n_{1}, m-m_{1}} \\
& +\bar{a}_{1102} \sum_{m_{1}=0}^{m} \sum_{m_{2}=0}^{m-m_{1}} \sum_{n_{1}=0}^{n} \sum_{n_{2}=0}^{n-n_{1}} \bar{\Gamma}_{m_{1}, n_{1}} \Delta_{m_{2}, n_{2}} \Delta_{m-m_{1}-m_{2}, n-n_{1}-n_{2}} \\
& \left.+\bar{a}_{1210} \sum_{m_{1}=0}^{m} \sum_{m_{2}=0}^{m-m_{1}} \sum_{n_{1}=0}^{n} \sum_{n_{2}=0}^{n-n_{1}} \bar{\Gamma}_{m_{1}, n_{1}} \bar{\Gamma}_{m_{2}, n_{2}} \Gamma_{n-n_{1}-n_{2}, m-m_{1}-m_{2}}\right)
\end{aligned}
$$




$$
\begin{aligned}
\Delta_{m, n}=\frac{1}{(m+n) \gamma-\lambda+i(m-n) \omega}( & a_{2002} \sum_{m_{1}=0}^{m} \sum_{n_{1}=0}^{n} \Delta_{m-m_{1}, n-n_{1}} \Delta_{m_{1}, n_{1}}+a_{2110} \sum_{m_{1}=0}^{m} \sum_{n_{1}=0}^{n} \Gamma_{m_{1}, n_{1}} \bar{\Gamma}_{n-n_{1}, m-m_{1}} \\
& +a_{2111} \sum_{m_{1}=0}^{m} \sum_{m_{2}=0}^{m-m_{1}} \sum_{n_{1}=0}^{n} \sum_{n_{2}=0}^{n-n_{1}} \Gamma_{m_{1}, n_{1}} \bar{\Gamma}_{m_{2}, n_{2}} \Delta_{m-m_{1}-m_{2}, n-n_{1}-n_{2}} \\
& \left.+a_{2003} \sum_{m_{1}=0}^{m} \sum_{m_{2}=0}^{m-m_{1}} \sum_{n_{1}=0}^{n} \sum_{n_{2}=0}^{n-n_{1}} \Delta_{m_{1}, n_{1}} \Delta_{m_{2}, n_{2}} \Delta_{m-m_{1}-m_{2}, n-n_{1}-n_{2}}\right) .
\end{aligned}
$$

These foregoing 1D and 2D invariant manifolds finally form the Shilnikov type principal homoclinic orbit $H$ in its analytical form. It benefits the construction of the second order Poincaré map shown in the Section 4.

\section{The Second Order Poincaré Map}

The Poincaré map is one of the most commonly used approach to study the dynamics surrounding the Shilnikov homoclinic loop including double or multipulse (circuit) orbits. In a general process, the first step to construct a return map is built from the composition of the first hit maps between the surfaces of section $[4,17]$. The first hit maps are locally defined since at $\mu=0$ the surfaces of the section intersect the homoclinic cycle transversally. So, with the obtained analytical manifolds, the local linear stable and unstable manifolds are given by

$$
\begin{aligned}
& u_{1}=\left(\Gamma_{r, 1,0}+i \Gamma_{i, 1,0}\right) e^{\lambda_{2} t}+o\left(e^{\lambda_{2} t}, e^{\lambda_{3} t}\right), \\
& \bar{u}_{1}=\left(\Gamma_{r, 1,0}-i \Gamma_{i, 1,0}\right) e^{\lambda_{3} t}+o\left(e^{\lambda_{2} t}, e^{\lambda_{3} t}\right), \\
& u_{2}=\Delta_{1} e^{\lambda t}+o\left(e^{\lambda t}\right) .
\end{aligned}
$$

The orbit $H$ intersects the complex plane $\left(\Gamma_{r, m, n}, \Gamma_{i, m, n}\right)$ at a countable set of points at $\mu=0$. So, we select $\left(\Gamma_{r, 1,0}+i \Gamma_{i, 1,0}, \Delta_{1}\right)$ as the starting point of $(8)$ in its coordinates and construct a two-dimensional cross-section $\Pi_{0}$ as shown in Figure 2. Following the orbit, there emerges another point $u_{1}^{\Pi_{1}}$ belonging to the second auxiliary cross-section $\Pi_{1}$. Let $\Pi_{0}$ be a rectangle lying in the plane $\left(\Gamma_{r, m, n}, \Gamma_{i, m, n}\right)$ and $\Pi_{1}$ a rectangle parallel to the plane at $u_{2}^{\Pi_{1}}=\Delta_{1} e^{\lambda T_{0}}=\varepsilon$. So, the Poincaré map $P=P_{0} \circ P_{1}$ along the orbit should be constructed by dividing the flow close to the stationary point of its unstable manifold into two parts: a small neighborhood of the stationary point where the flow is essentially linear and a global flow which takes trajectories close to the unstable manifold away from and then back to the linear region, as shown in Figure 1.

Starting from the point $\left(\Gamma_{r, 1,0}+i \Gamma_{i, 1,0}, 0\right)$ on $\Pi_{0}$ to reach $(0, \varepsilon)$ on $\Pi_{1}$, it produces the first local map $P_{0}: \Pi_{0} \rightarrow \Pi_{1}$. According to (8), this local map can be written as

$$
P_{0}: \Pi_{0} \longrightarrow \Pi_{1}: u_{1}^{\Pi_{1}}=\left(\Gamma_{r, 1,0}+i \Gamma_{i, 1,0}\right) e^{\lambda_{2} T_{0}}+o\left(e^{\lambda_{2} T_{0}}, e^{\lambda_{3} T_{0}}\right) .
$$

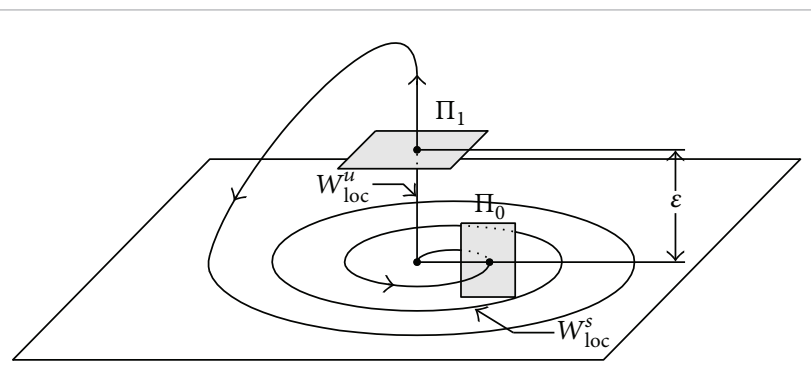

Figure 1: Cross-sections $\Pi_{0}$ and $\Pi_{1}$ near the origin.

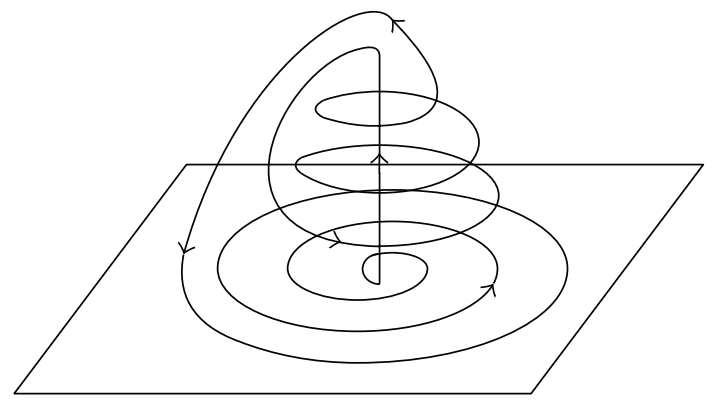

FIgURE 2: Subsidiary homoclinic orbit.

From the equation of $u_{2}$, the time $T_{0}$ of flight for points starting on $\Pi_{0}$ to reach $\Pi_{1}$ can be calculated from

$$
T_{0}=-\frac{1}{\lambda} \log \left(\frac{\Delta_{1}}{\varepsilon}\right) .
$$

Substituting the flight time into map $P_{0}$, it is found that the expression for the first hit map can be expressed as

$$
P_{0}: \Pi_{0} \longrightarrow \Pi_{1}: u_{1}^{\Pi_{1}}=\left(\Gamma_{r, 1,0}+i \Gamma_{i, 1,0}\right) u^{\rho}+o\left(e^{\lambda_{2} T_{0}}, e^{\lambda_{3} T_{0}}\right),
$$

where $u=\Delta_{1} / \varepsilon, \rho=-\lambda_{2} / \lambda=\rho_{r}+i \rho_{i}$. Since the time of flight $t_{0}$ is bounded in this progress, which generates another global map according to (6),

$$
P_{1}: \Pi_{1} \longrightarrow \Pi_{0}:\left\{\begin{array}{c}
\Gamma_{r, 1,0}+i \Gamma_{i, 1,0}-(x+y i) \\
=u_{1}^{\Pi_{1}} e^{\lambda_{2} t_{0}}+f_{1}\left(e^{\lambda_{2} t_{0}}, e^{\lambda_{3} t_{0}}\right), \\
\Delta_{1}-\mu \\
=\Delta_{1,0} u_{1}^{\Pi_{1}} e^{\lambda_{2} t_{0}}+\Delta_{0,1} \bar{u}_{1}^{\Pi_{1}} e^{\lambda_{3} t_{0}} \\
\quad+f_{2}\left(e^{\lambda_{2} t_{0}}, e^{\lambda_{3} t_{0}}\right),
\end{array}\right.
$$


where $f_{1}, f_{2}$ include the remaining components of $e^{\lambda_{2} t_{0}}, e^{\lambda_{3} t_{0}}$, and these equations portrait the global behavior outside the equilibrium. So, the combination of the local and global map finally forms the second order Poincaré map:

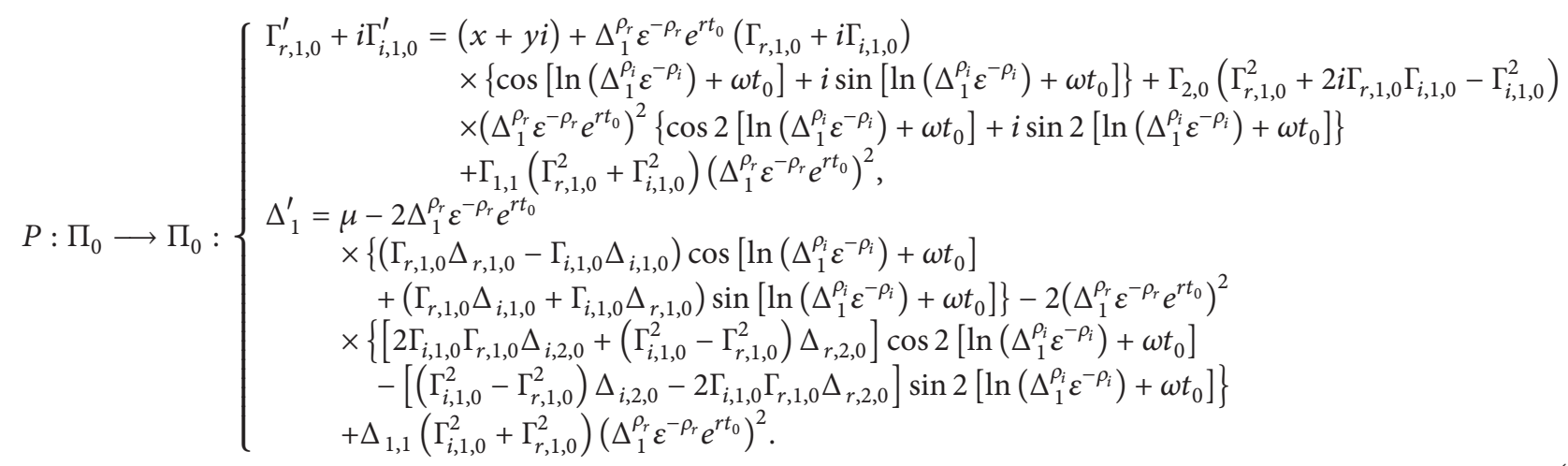

That is a more general expression of the return map because the former results

$$
P: \Pi_{0} \longrightarrow \Pi_{0}:\left\{\begin{array}{c}
\Gamma_{i, 1,0}^{\prime}=1+\Delta_{1}^{\rho_{r}} \Gamma_{i, 1,0} \cos \left(\ln \Delta_{1}^{\rho_{i}}+\theta\right) \\
\Delta_{1}^{\prime}=\mu+\Delta_{1}^{\rho_{r}} \Gamma_{i, 1,0} \\
\times\left[\Delta_{i, 1,0} \cos \left(\ln \Delta_{1}^{\rho_{i}}+\theta\right)\right. \\
\left.-\Delta_{r, 1,0} \sin \left(\ln \Delta_{1}^{\rho_{i}}+\theta\right)\right]
\end{array}\right.
$$

as given by Gonchenko et al. and Zhou et al. [6, 7], can be easily obtained by letting $\varepsilon=1, x=0, y=1, \Gamma_{r, 1,0}=$ $0, \omega t_{0}=\theta, \Delta_{1}^{\rho_{r}} e^{r t_{0}} \approx \Delta_{1}^{\rho_{r}}, 2 \Delta_{i, 1,0}=\Delta_{i, 1,0}, 2 \Delta_{r, 1,0}=\Delta_{r, 1,0}$, and ignoring the second order components, while the new map makes itself in a broader usage because of the general expression and better computational precision.

\section{Conclusions}

In this paper, the second order Poincaré map in a common 3D nonlinear system is constructed. Shilnikov sense subsidiary homoclinic dynamics is also researched. Firstly, we find the strategy to analytically construct the homoclinic orbit surrounding the saddle-focus equilibrium point in terms of the complex SNF and method of invariant manifolds. By using these quantified manifolds, we can form the expression of the first and the second order Poincare map, making their appearance in a more general style with the capacity to enhance computational precise. The further possible application of the subsidiary homoclinicity is shown in Figure 2 which is a diagram for subsidiary homoclinic orbit; the return map can portrait the dynamics unfolding from the principal orbit.

However, the analytical work lags behind the numerical results in great extent. So, to employ the higher order Poincaré map and then to unfold the successive bifurcation behaviors more simply and exactly, in a narrow parameter interval, will be the topics for further discussion.

\section{Appendix}

The SNF coefficients of the Hopf-zero bifurcation up to the third order are as follows:

$$
\begin{aligned}
b_{1,1,0,1}= & a_{1,1,0,1} ; \quad b_{2,0,0,2}=a_{2,0,0,2} ; \\
b_{2,1,1,0}= & a_{2,1,1,0} ; \quad b_{2,0,0,3}=a_{2,0,0,3} ; \\
b_{1,2,1,0}= & -\frac{a_{2,1,1,1} a_{1,1,1,0,1}}{2\left(a_{2,0,0,2}-a_{1,1,1,0,1}\right)} \\
& -\frac{a_{2,1,1,0} a_{1,1,1,0,2}}{a_{2,0,0,2}-a_{1,1,1,0,1}}+a_{1,1,2,1,0} \\
& +i\left(-\frac{a_{2,1,1,1} a_{2,1,1,0,1}}{2\left(a_{2,0,0,2}-a_{1,1,1,0,1}\right)}+\frac{a_{2,1,1,0} a_{1,1,1,0,2} a_{2,1,1,0,1}}{a_{2,0,0,2} a_{1,1,1,0,1}}\right. \\
& \quad-\frac{a_{2,1,1,0} a_{1,1,1,0,2} a_{2,1,1,0,1}}{\left(a_{2,0,0,2}-a_{1,1,1,0,1}\right) a_{1,1,1,0,1}} \\
& \left.\quad-\frac{a_{2,1,1,0} a_{2,1,1,0,2}}{a_{2,0,0,2}}+a_{2,1,2,1,0}\right) .
\end{aligned}
$$

The second order coefficients of the two-dimensional manifold are as follows

$$
\begin{aligned}
& \Gamma_{2,0}=\frac{b_{1101} \Delta_{1,0}}{\gamma+i \omega}, \quad \bar{\Gamma}_{2,0}=\frac{\bar{b}_{1101} \Delta_{0,1}}{\gamma-i \omega}, \quad \Gamma_{1,1}=\frac{b_{1101} \Delta_{0,1}}{\gamma-i \omega}, \\
& \bar{\Gamma}_{1,1}=\frac{\bar{b}_{1101} \Delta_{1,0}}{\gamma+i \omega}, \quad \Gamma_{0,2}=0, \quad \bar{\Gamma}_{0,2}=0, \\
& \Delta_{2,0}=\frac{b_{2002} \Delta_{1,0}^{2}}{2 \gamma-\lambda+2 i \omega}, \quad \Delta_{0,2}=\frac{b_{2002} \Delta_{0,1}^{2}}{2 \gamma-\lambda-2 i \omega}, \\
& \Delta_{1,1}=\frac{b_{2110}+2 b_{2002} \Delta_{0,1} \Delta_{1,0}}{2 \gamma-\lambda} .
\end{aligned}
$$




\section{Conflict of Interests}

The authors declare that there is no conflict of interests.

\section{Acknowledgments}

This work was supported by the National Natural Science Foundation of China (Grant no. 11272229) and Science Foundation of Tianjin education committee (Grant no. 20120902).

\section{References}

[1] M. Ferrara, C. Bianca, and L. Guerrini, "High-order moments conservation in thermostatted kinetic models," Journal of Global Optimization, 2013.

[2] M. Ferrara, F. Munteanu, C. Udrişte, and D. Zugrăvescu, "Controllability of a nonholonomic macroeconomic system," Journal of Optimization Theory and Applications, vol. 154, no. 3, pp. 1036-1054, 2012.

[3] M. Ferrara and C. Udrişte, "Multitime models of optimal growth," WSEAS Transactions on Mathematics, vol. 7, no. 1, pp. 51-55, 2008.

[4] Y. A. Kuznetsov, Elements of Applied Bifurcation Theory, vol. 112 of Applied Mathematical Sciences, Springer, New York, NY, USA, 2nd edition, 1998.

[5] P. Glendinning and C. Sparrow, "Local and global behavior near homoclinic orbits," Journal of Statistical Physics, vol. 35, no. 5-6, pp. 645-696, 1984.

[6] S. V. Gonchenko, D. V. Turaev, P. Gaspard, and G. Nicolis, "Complexity in the bifurcation structure of homoclinic loops to a saddle-focus," Nonlinearity, vol. 10, no. 2, pp. 409-423, 1997.

[7] T. S. Zhou, G. R. Chen, and Q. G. Yang, "Constructing a new chaotic system based on the Šilnikov criterion," Chaos, Solitons and Fractals, vol. 19, no. 4, pp. 985-993, 2004.

[8] Z. Li, G. R. Chen, and W. A. Halang, "Homoclinic and heteroclinic orbits in a modified Lorenz system," Information Sciences, vol. 165, no. 3-4, pp. 235-245, 2004.

[9] Q. C. Zhang, R. L. Tian, and W. Wang, "Chaotic properties of mechanically and electrically coupled nonlinear dynamical systems," Acta Physica Sinica, vol. 57, no. 5, pp. 2799-2804, 2008.

[10] Y.H. Li and S.M. Zhu, "N-dimensional stable and unstable manifolds of hyperbolic singular point," Chaos, Solitons and Fractals, vol. 29, no. 5, pp. 1155-1164, 2006.

[11] I. Baldomá and T. M. Seara, "Breakdown of heteroclinic orbits for some analytic unfoldings of the Hopf-zero singularity," Journal of Nonlinear Science, vol. 16, no. 6, pp. 543-582, 2006.

[12] P. Yu and A. Y. T. Leung, "A perturbation method for computing the simplest normal forms of dynamical systems," Journal of Sound and Vibration, vol. 261, no. 1, pp. 123-151, 2003.

[13] Q. C. Zhang and W. Wang, "Simplest normal form for the singularity of a pair of pure imaginary and a zero eigenvalue system," Tianjin Daxue Xuebao, vol. 40, pp. 971-975, 2007.

[14] W. Wang and Q.C. Zhang, "Computation of the simplest normal form of a resonant double Hopf bifurcation system with the complex normal form method," Nonlinear Dynamics, vol. 57, no. 1-2, pp. 219-229, 2009.

[15] A. H. Nayfeh, Method of Normal Forms, Wiley Series in Nonlinear Science, John Wiley \& Sons, New York, NY, USA, 1993.

[16] S. Wiggins, Global Bifurcations and Chaos, vol. 73 of Applied Mathematical Sciences, Springer, New York, NY, USA, 1988.
[17] F. S. Cui, C. H. Chew, J. X. Xu, and Y. L. Cai, "Bifurcation and chaos in the Duffing oscillator with a PID controller," Nonlinear Dynamics, vol. 12, no. 3, pp. 251-262, 1997. 


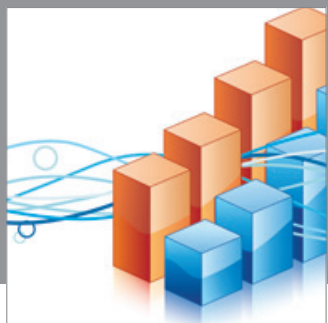

Advances in

Operations Research

mansans

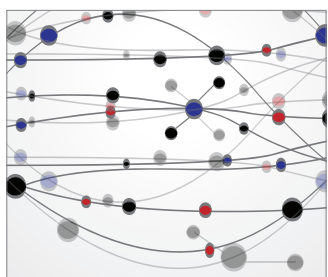

The Scientific World Journal
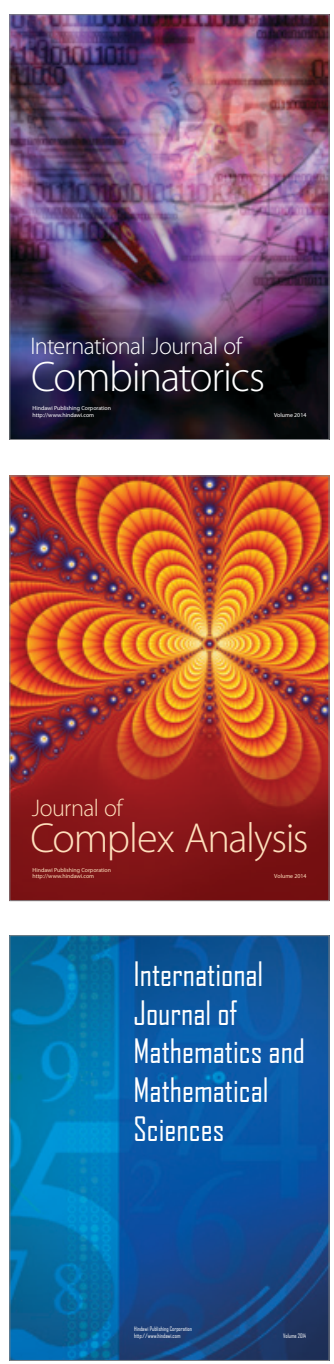
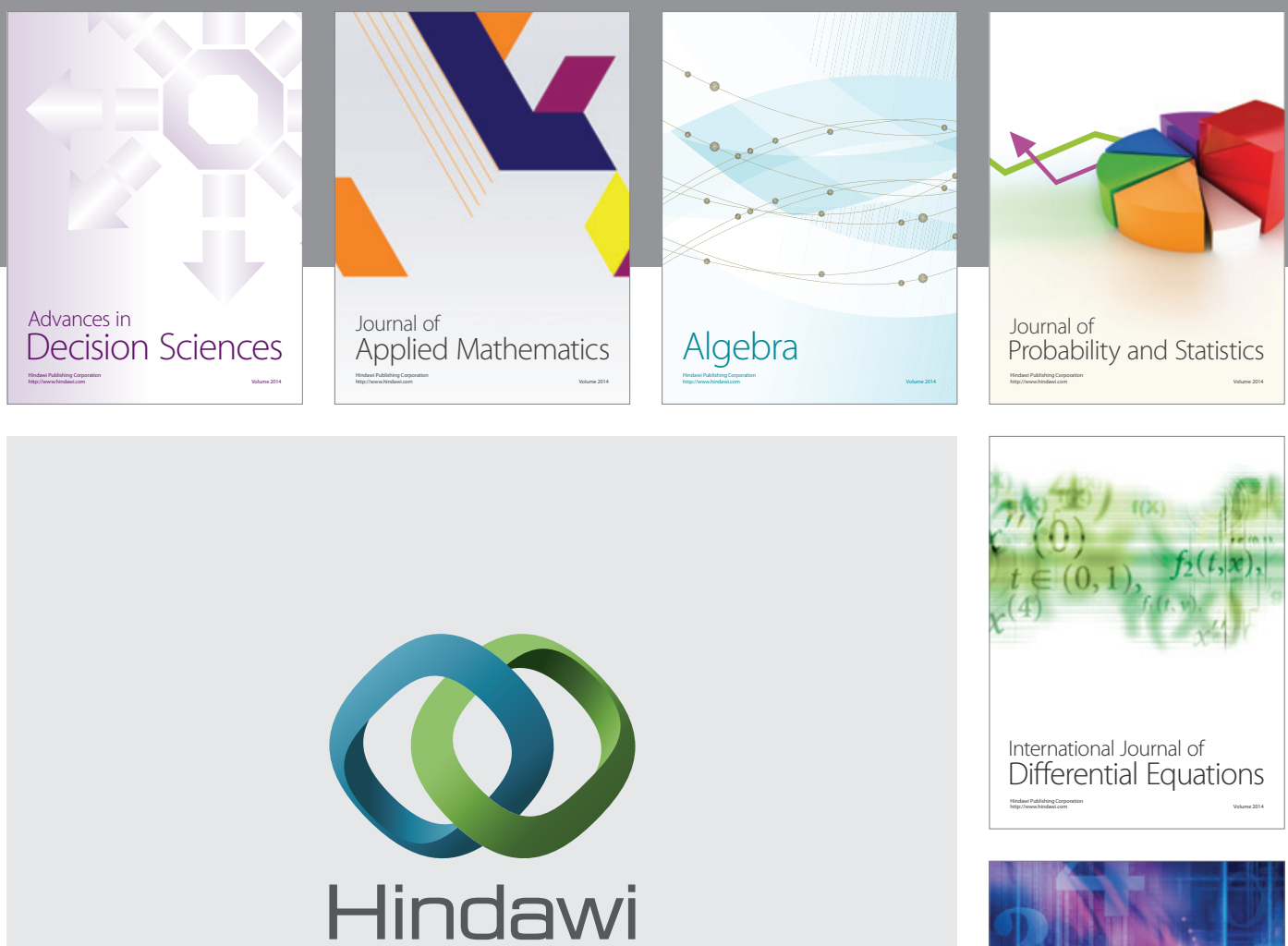

Submit your manuscripts at http://www.hindawi.com
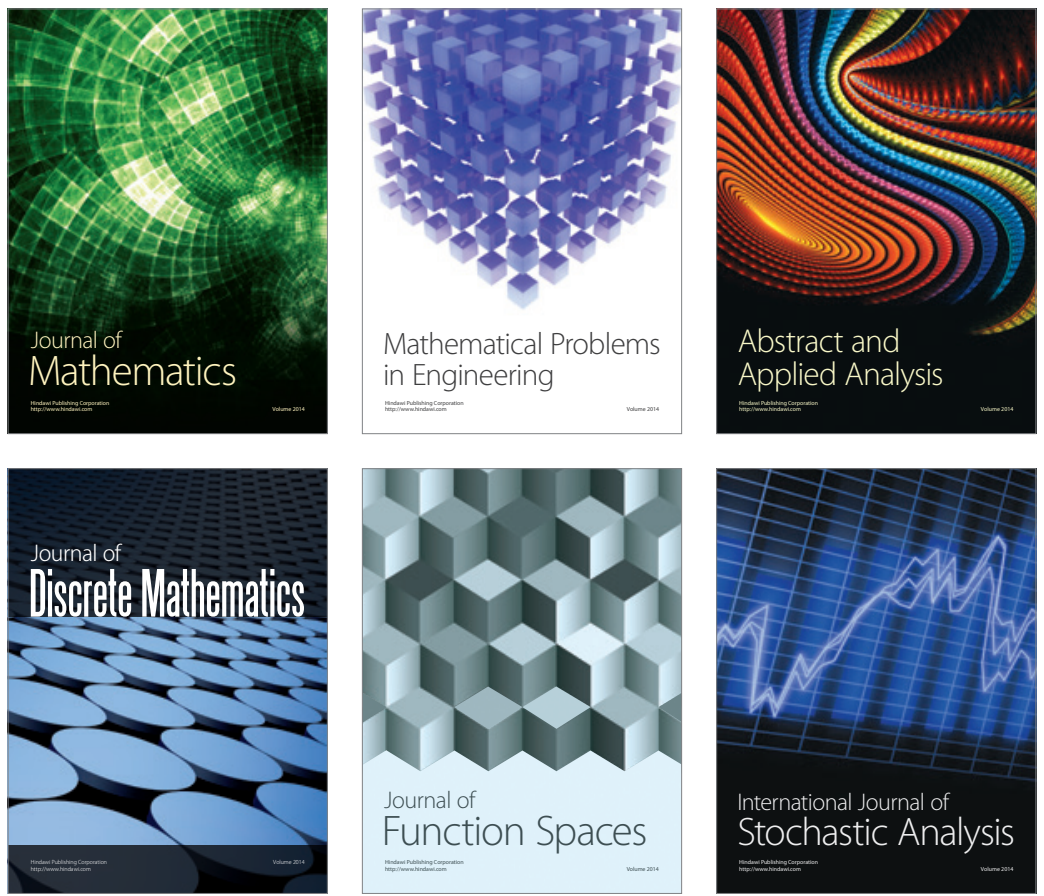

Journal of

Function Spaces

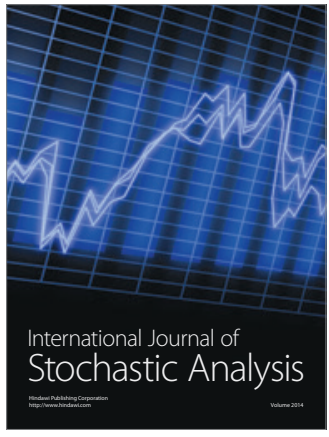

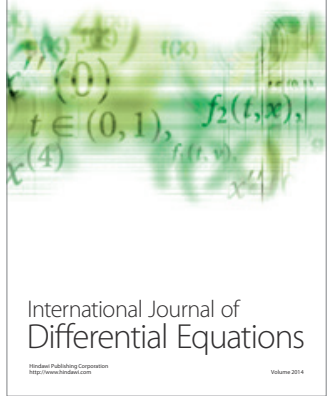
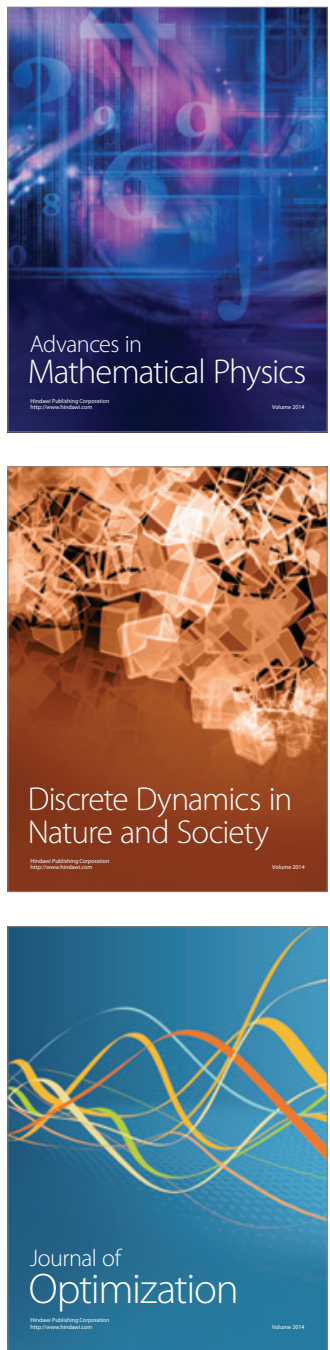